\title{
Energy expenditure assessed by heart rate and doubly labeled water in young athletes
}

Citation for published version (APA):

Ekelund, U., Yngve, A., Westerterp, K. R., \& Sjostrom, M. (2002). Energy expenditure assessed by heart rate and doubly labeled water in young athletes. Medicine and Science in Sports and Exercise, 34(8), 1360-1366. https://doi.org/10.1097/00005768-200208000-00019

Document status and date:

Published: 01/01/2002

DOI:

10.1097/00005768-200208000-00019

Document Version:

Publisher's PDF, also known as Version of record

Document license:

Taverne

Please check the document version of this publication:

- A submitted manuscript is the version of the article upon submission and before peer-review. There can be important differences between the submitted version and the official published version of record.

People interested in the research are advised to contact the author for the final version of the publication, or visit the DOI to the publisher's website.

- The final author version and the galley proof are versions of the publication after peer review.

- The final published version features the final layout of the paper including the volume, issue and page numbers.

Link to publication

\footnotetext{
General rights rights.

- You may freely distribute the URL identifying the publication in the public portal. please follow below link for the End User Agreement:

www.umlib.nl/taverne-license

Take down policy

If you believe that this document breaches copyright please contact us at:

repository@maastrichtuniversity.nl

providing details and we will investigate your claim.
}

Copyright and moral rights for the publications made accessible in the public portal are retained by the authors and/or other copyright owners and it is a condition of accessing publications that users recognise and abide by the legal requirements associated with these

- Users may download and print one copy of any publication from the public portal for the purpose of private study or research.

- You may not further distribute the material or use it for any profit-making activity or commercial gain

If the publication is distributed under the terms of Article $25 \mathrm{fa}$ of the Dutch Copyright Act, indicated by the "Taverne" license above, 


\title{
Energy expenditure assessed by heart rate and doubly labeled water in young athletes
}

\author{
ULF EKELUND, AGNETA YNGVE, KLAAS WESTERTERP, and MICHAEL SJÖSTRÖM \\ Unit for Preventive Nutrition at Novum, Department of Medical Nutrition/Biosciences, Karolinska Institutet, Stockholm, \\ SWEDEN; Department of Physical Education and Health, Örebro University, SWEDEN; and Department of Human \\ Biology, Maastricht University, Maastricht, THE NETHERLANDS
}

\begin{abstract}
EKELUND, U., A. YNGVE, K. WESTERTERP, and M. SJÖSTRÖM. Energy expenditure assessed by heart rate and doubly labeled water in young athletes. Med. Sci. Sports Exerc., Vol. 34, No. 8, pp. 1360-1366, 2002. Purpose: To compare total energy expenditure (TEE) estimated by the FLEX heart rate (HR) method with that measured by the doubly labeled water (DLW) technique in young speed skaters. We hypothesized that the accuracy of FLEX HR-estimated TEE would be affected by a) the definition of the FLEX HR and b) the type of training regimen. Methods: Eight young athletes (mean age $18.2 \pm 1.3 \mathrm{yr}$ ) underwent measurements during two 10-d training periods: an off-season period with voluntary training (predominantly running) and a preseason period mainly focused on skating technique training. TEE was measured simultaneously by the DLW and FLEX HR methods. FLEX HR1 was defined as the mean of the HRs during all resting calibration activities and the lowest HR during exercising calibration activities. FLEX HR2 was defined as the mean of the highest HR during resting activities and the lowest HR during exercising. Results: ANOVA showed that FLEX HR1 was significantly lower than FLEX HR2 (mean of both periods; $77 \pm 5$ vs $84 \pm 6$ beats $\cdot \min ^{-1} ; P=0.004$ ). TEE values obtained by DLW were $16.8 \pm 3.8$ and $16.9 \pm 2.9 \mathrm{MJ} \cdot \mathrm{d}^{-1}$ in the two periods, respectively. TEE values calculated from FLEX HR1 were $17.8 \pm 3.6$ and $17.4 \pm 2.6 \mathrm{MJ} \cdot \mathrm{d}^{-1}$, and those from FLEX HR2 $17.1 \pm 3.1$ and $17.0 \pm 2.7 \mathrm{MJ} \cdot \mathrm{d}^{-1}$, respectively. No significant period $(P=0.83$ ) or method $(P=0.44)$ effect on TEE was observed. Conclusion: FLEX HR-estimated TEE was not affected by the definition of the FLEX HR or by the type of training regimen as compared with TEE measured by the DLW method in young athletes. Key Words: FLEX HEART RATE, TOTAL ENERGY EXPENDITURE, SPEED SKATERS, PHYSICAL ACTIVITY
\end{abstract}

A

thletic training and competition usually require high energy expenditure, which has to be balanced by an equally high energy intake to achieve energy balance. However, the current knowledge of the energy turnover in athletes is limited $(1,18)$. This is at least partly due to the difficulties in accurately assessing the free-living patterns of energy expenditure. Besides the doubly labeled water (DLW) method, which is currently regarded as the "gold standard" for measurement of total daily energy expenditure (TEE) under free-living conditions, a number of different assessment techniques have been developed.

One of these, minute-by-minute heart rate (HR) monitoring according to the FLEX HR method (17) has previously been used to assess TEE and associated patterns of physical activity in athletes (4). Although the FLEX HR method has been extensively validated against the DLW technique in several groups of subjects, such as children with various levels of physical activity $(10,19)$, obese children (14), farmers involved in heavy manual labor (8), and overweight women (6), the conformity between TEE estimates obtained by these two methods in young athletes remains to be evaluated.

0195-9131/02/3408-1360/\$3.00/0

MEDICINE \& SCIENCE IN SPORTS \& EXERCISE ${ }_{\circledast}$

Copyright $\odot 2002$ by the American College of Sports Medicine

Submitted for publication September 2001.

Accepted for publication March 2002.
There are at least two key factors that might have an impact on the validity and accuracy of the FLEX HR method. One is the definition of the FLEX HR. The FLEX $\mathrm{HR}$ is an individually predetermined HR, which is used to discriminate between rest and physical activity during the monitoring period. Normally, the FLEX HR is defined as the mean of the highest HR during rest and the lowest HR during light exercise (11). However, there is no consensus today on the most appropriate way of defining the FLEX HR, and different definitions have been proposed $(6,8,10,11)$. It seems from previous reports that the accuracy of TEE estimates is affected by the definition of the FLEX $\mathrm{HR}$, at least in overweight women (6). However, this line of investigation needs to be followed up further in other groups of subjects with various levels of physical activity.

Second, although the FLEX HR method relies on individual calibration of the relationship between HR and oxygen uptake $\left(\mathrm{VO}_{2}\right)$ during activities of various types and intensities in the laboratory, the representativeness of the derived $\mathrm{HR}-\dot{\mathrm{V}}_{2}$ regression equation for free-living situations may have an impact on the accuracy. It has been argued that the number and type of calibration activities may influence the accuracy of the method $(5,9)$. On the other hand, Livingstone et al. (12) did not observe any differences in TEE calculated from seven different calibration protocols in young boys. In line with this, another issue that remains to be solved is whether the limited calibration activities are representative of the various types of exercises that are often carried out by athletes during different training regimens. 
The purpose of the present study was to compare estimates of TEE obtained by the FLEX HR method with TEE as measured by the DLW technique in young athletes. We hypothesized that the accuracy of the FLEX HR method would be affected a) by the definition of the FLEX HR and b) by the type of training regimen.

\section{METHODS}

Subjects. Eight adolescent male athletes (speed skaters) volunteered to participate (Table 1). All had been engaged in regular speed skating training for at least $4 \mathrm{yr}$, and all were living at a boarding school for young athletes. Written informed consent was obtained from all subjects and from the parents of those who were younger than $18 \mathrm{yr}$ of age. The experimental protocol was approved by the ethics committee of Karolinska Institute, Stockholm, Sweden.

Experimental design. All measurements were performed in exactly the same way during two different training periods. The first period was a 10-d off-season period and the second one was a 10-d preseason-training period. During the off-season period, all physical training was voluntary, and the main exercise performed by the subjects was jogging or running. The preseason period, starting 5 months after the off-season period, was mainly focused on training of skating techniques. During this period, the exercises included weight training, in-line skating, slideboard training, circuit training, and skating imitations. Thus, none of these periods were focused on high-intensity aerobic training aimed to increase $\mathrm{VO}_{2 \max }$. Differences in the type and duration of exercises between periods were checked by scrutinizing the subjects' training diaries. These were subsequently reevaluated through personal communication with the coach.

TEE was measured by the DLW method and estimated by the FLEX HR method over the two 10-d periods. The HR monitors were worn on day 1 through 8 . Both periods were preceded by individual calibration to establish the HRenergy expenditure (EE) relationship during standardized activities. Fasting body weight was measured in the morning on days 1 and 10, to investigate energy balance.

Body composition and maximal oxygen uptake. Height was measured with a wall-mounted stadiometer to the nearest $0.5 \mathrm{~cm}$. Body weight was measured after an overnight fast (days 1 and 10) in light underwear, using an electronic scale, to the nearest $0.1 \mathrm{~kg}$. Total body water
(TBW) was assessed by deuterium dilution (20). The fatfree body weight (fat-free mass, FFM) was calculated from TBW assuming a hydration factor for FFM of 0.732 (15). Body fat $(\%)$ was calculated as $100 \times$ (body weight FFM)/body weight.

All laboratory measurements were performed at the subject's school. Maximal oxygen uptake $\left(\mathrm{V}_{2 \max }\right)$ was determined during an incremental test on a mechanically braked cycle ergometer (Monark 818E, Monark AB, Varberg, Sweden). The test was initiated by two 6-min steady-state workloads at $120 \mathrm{~W}$ and $180 \mathrm{~W}$. The workload was then increased by $25 \mathrm{~W} \cdot \mathrm{min}^{-1}$ to exhaustion. Ventilation, oxygen uptake $\left(\dot{\mathrm{VO}}_{2}\right)$, and carbon-dioxide $\left(\mathrm{CO}_{2}\right)$ production were measured at 15-s intervals using an on-line lightweight portable telemetric system, Cosmed K4 (Cosmed Srl., Rome, Italy). The system was calibrated against gases of known concentrations before testing and the flowmeter was calibrated against a 3.0-L syringe (Hans Rudolph Inc., Kansas City, MO). Calibration procedures described by the manufacturer were otherwise followed. The portable ergospirometer system used has been validated and has shown accurate readings for oxygen uptake measurements from sitting at rest to maximal exercise (7). HR was measured simultaneously using a telemetric system (Polar Vantage, Polar Electro OY, Kempele, Finland).

Assessment of total energy expenditure. The criterion TEE was measured by the DLW method as described by Westerterp et al. (20). Briefly, the evening before day 1 of the 10-d period, the subject drank an individually weighed dose of ${ }^{18} \mathrm{O}$ and ${ }^{2} \mathrm{H}$ after providing a baseline urine sample. The dose of the isotopes was calculated to lead to an initial excess body water enrichment of $150 \mathrm{ppm}$ for ${ }^{2} \mathrm{H}$ and $300 \mathrm{ppm}$ for ${ }^{18} \mathrm{O}$. Urine samples were then collected from the second and last voidings on days 1, 5, and 10. The isotope quantities in the urine samples were measured with an isotope-ratio mass spectrometer (Aqua Sira; VG Isogas, Middlewich, Cheshire, UK). All samples were measured in duplicate. $\mathrm{CO}_{2}$ production was derived from the elimination rates of the isotopes, as calculated from the slope of the elimination curve, with correction for changes in body water, which were assumed to be proportional to changes in body mass, from day 1 to $10 . \mathrm{CO}_{2}$ production was converted to TEE using an energy equivalent based on the individual food quotient (FQ) calculated from the macronutrient composition of the diet (2).

TABLE 1. Characteristics of the subjects.

\begin{tabular}{|c|c|c|c|c|c|c|}
\hline & \multicolumn{3}{|c|}{ Off-Season } & \multicolumn{3}{|c|}{ Preseason } \\
\hline & Mean \pm SD & Min & Max & Mean \pm SD & Min & Max \\
\hline $\begin{array}{l}\text { Age }(\mathrm{yr}) \\
\text { Height }(\mathrm{m}) \\
\text { Weight }(\mathrm{kg}) \\
\text { Fat-free mass }(\mathrm{kg}) \\
\text { Fat (\% body weight) } \\
10-\mathrm{d} \text { weight change }(\mathrm{kg})^{a} \\
\mathrm{~V} 0_{2 \max }\left(\mathrm{mL} \cdot \mathrm{kg}^{-1} \cdot \mathrm{min}^{-1}\right)\end{array}$ & $\begin{aligned} & 18.2 \pm 1.3 \\
& 1.79 \pm 0.03 \\
& 75.7 \pm 7.0 \\
& 66.0 \pm 6.9 \\
& 12.8 \pm 3.0 \\
& 0.3 \pm 0.6 \\
& 64.0 \pm 2.5 \\
&\end{aligned}$ & $\begin{array}{c}16.5 \\
1.75 \\
70.4 \\
59.2 \\
8.6 \\
-0.4 \\
59.1 \\
\end{array}$ & $\begin{array}{c}20.3 \\
1.85 \\
92.3 \\
82.2 \\
17.7 \\
1.5 \\
66.8 \\
\end{array}$ & $\begin{array}{l}18.6 \pm 1.3 \\
1.80 \pm 0.03 \\
76.4 \pm 6.6 \\
67.0 \pm 5.8 \\
12.2 \pm 3.0 \\
0.05 \pm 0.6 \\
67.7 \pm 4.1^{*}\end{array}$ & $\begin{array}{c}17.0 \\
1.75 \\
71.4 \\
60.9 \\
7.1 \\
-0.6 \\
60.9 \\
\end{array}$ & $\begin{array}{c}20.8 \\
1.85 \\
91.3 \\
80.0 \\
14.7 \\
1.2 \\
73.4 \\
\end{array}$ \\
\hline
\end{tabular}

${ }^{a}$ Weight change during the doubly labeled water measurement period.

${ }^{*} P<0.05$. 
Energy intake (EI) was assessed from a weighed food record during the first $7 \mathrm{~d}$ of the measurement periods to derive the individual FQ. All food consumed (i.e., at home, at school, and in restaurants) was weighed on a household scale and recorded. EI was calculated from the food records by means of a computerized nutrient calculation software using the Swedish nutrient database (National Food Administration).

HR monitoring. The subject's HR was monitored minute-by-minute from day 1 to 8 , including two weekend days and six weekdays, during the time of the day in which the subjects were awake. A telemetric HR monitor (Polar Vantage) was used. The mean monitoring times, in the two periods were $15.3 \pm 0.7$ and $15.2 \pm 0.7 \mathrm{~h} \cdot \mathrm{d}^{-1}$, respectively. Unreliable HR values, i.e., spikes above maximal HR and HRs below 35 bpm, were eliminated before analysis.

Each subject underwent individual calibrations to establish the relationship between $\mathrm{HR}$ and $\mathrm{V}_{2}$. This calibration procedure was carried out during the daytime, $3 \mathrm{~h}$ on average, after the last meal. The following activities were performed for determination of the individual relationship between $\dot{\mathrm{VO}}_{2}$ and HR: lying supine, sitting, standing, treadmill walking at 4.5 and $6.5 \mathrm{~km} \cdot \mathrm{h}^{-1}$, treadmill running at 10 $\mathrm{km} \cdot \mathrm{h}^{-1}$, and ergometer bicycling at 120 and $180 \mathrm{~W}$. The calibration activities were carried out in sequence with a break to allow recovery of resting HR between the treadmill and bicycle exercises. $\mathrm{HR}$ and $\mathrm{VO}_{2}$ were measured at 15 -s intervals, using the same system as described above. The gas sampling time was $6 \mathrm{~min}$, and calibration points were calculated as the mean $\mathrm{HR}$ and $\mathrm{VO}_{2}$ for the last $3 \mathrm{~min}$.

TEE was determined from minute-by-minute HR monitoring, using the HR FLEX method (17). Two different FLEX HRs were calculated: a "low" FLEX HR (FLEX HR1) calculated as the mean of the HRs during all resting calibration activities (i.e., supine, sitting, and standing) and the lowest HR during exercising (i.e., walking at 4.5 $\mathrm{km} \cdot \mathrm{h}^{-1}$ ), and a "high" FLEX HR (FLEX HR2), calculated as the mean of the highest HR during the resting activities (i.e., standing) and the lowest HR during exercising (i.e., walking at $4.5 \mathrm{~km} \cdot \mathrm{h}^{-1}$ ). The HR and EE data above FLEX HR were used to calculate individual linear regressions (EE $=$ constant + slope $\times$ HR). EE was calculated from the regression at all time points with HR $>$ FLEX HR. When HR was $\leq$ FLEX HR, EE was assumed to be the mean energy expended while supine, sitting, and standing and referred to as resting energy expenditure (REE). EE during sleep was assumed to be equal to the predicted basal metabolic rate (BMR) (16). TEE was obtained by summing the BMR, REE, and EE in activity ( $=$ HR $>$ FLEX). TEE from minute-by-minute HR monitoring using the low FLEX HR is referred to as TEEFLEX HR1 and TEE based on the high FLEX HR is referred to as TEEFLEX HR2.

The time $\left(\mathrm{min} \cdot \mathrm{d}^{-1}\right)$ spent in physical activity was calculated on the basis of FLEX HR1 and FLEX HR2 and averaged over the 8-d period. Individual HRs corresponding to $50 \%$ and $70 \%$ of $\dot{\mathrm{VO}}_{2 \max }$ were calculated from calibration data, and the average time $\left(\mathrm{min} \cdot \mathrm{d}^{-1}\right)$ spent at these intensity levels was calculated.

Activity energy expenditure and physical activity level. The energy expenditure associated with physical activity (AEE) was calculated as TEE (from DLW) * $0.9-$ BMR, with correction for a $10 \%$ diet-induced thermogenesis (13). The physical activity level (PAL) was expressed as the ratio of TEE (from DLW) to BMR.

Data analyses. Differences between periods (off-season and preseason) in physical characteristic variables, physiological data, DLW-measured energy expenditure, HRs (FLEX HR1, FLEX HR2, and daytime HR), and time spent at $50 \%$ and $70 \%$ of $\mathrm{VO}_{2 \max }$ were tested by analysis of variance (ANOVA). The effects of period (off-season and preseason) and method (FLEX HR1 and FLEX HR2) on the calculated time spent in physical activity ( $>$ FLEX HR) and the effects of period and method (FLEX HR1, FLEX HR2, and DLW) on the estimated TEE were tested by a three-way ANOVA where subject was considered as a random factor and period and method as fixed factors. All assumptions for ANOVA were fulfilled and the residuals showed a satisfactory pattern. The degree of agreement between TEE measured by DLW and TEE estimated from FLEX HR1 and FLEX HR2 was determined using the method described by Bland and Altman (3). Intermethod bias was defined as the mean difference in TEE between the criterion method (DLW) and the alternative methods (TEEFLEX HR1 and TEEFLEX HR2). The level of statistical significance was set as $P<0.05$. SPSS (Statistical Package for the Social Sciences for Windows, 8.0, 1997, SPSS Inc., Chicago. IL) was used for all statistical analyses.

\section{RESULTS}

Table 1 summarizes the physical characteristics of the subjects. There were no differences in any of the body composition variables between the two periods. Neither did the periods show a significant difference in weight change over the $10 \mathrm{~d}$. Mean maximal oxygen uptake was significantly higher during the preseason period than in the offseason period $(P<0.05)$. All subjects showed higher individual $\dot{\mathrm{VO}}_{2 \max }$ values during the preseason period than

TABLE 2. Daily energy expenditure estimates during the two different training periods in young athletes.

\begin{tabular}{|c|c|c|c|c|c|c|}
\hline & \multicolumn{3}{|c|}{ Off-Season } & \multicolumn{3}{|c|}{ Preseason } \\
\hline & Mean \pm SD & Min & $\operatorname{Max}$ & Mean \pm SD & Min & Max \\
\hline $\begin{array}{l}\text { BMR }\left(M J \cdot d^{-1}\right) \\
\text { TEE DLW }\left(M J \cdot d^{-1}\right) \\
\text { AEE }\left(M J \cdot d^{-1}\right) \\
\text { PAL }\end{array}$ & $\begin{array}{r}8.3 \pm 0.5 \\
16.8 \pm 3.8 \\
6.8 \pm 3.1 \\
2.0 \pm 0.4\end{array}$ & $\begin{array}{c}8.0 \\
12.8 \\
3.4 \\
1.58\end{array}$ & $\begin{array}{c}9.8 \\
25.0 \\
13.0 \\
2.63\end{array}$ & $\begin{array}{r}8.4 \pm 0.5 \\
16.9 \pm 2.9 \\
6.9 \pm 2.3 \\
2.0 \pm 0.2\end{array}$ & $\begin{array}{c}8.1 \\
13.9 \\
4.0 \\
1.62\end{array}$ & $\begin{array}{r}9.5 \\
23.8 \\
12.0 \\
2.52\end{array}$ \\
\hline
\end{tabular}

BMR, basal metabolic rate (estimated from ref. 15); TEE DLW, total energy expenditure, measured by the doubly labeled water method; AEE, activity energy expenditure (AEE = TEE $\mathrm{DLW} * 0.9$ - BMR); PAL, physical activity level (TEE/BMR). 
TABLE 3. Heart rates $\left(\mathrm{HR}\right.$; beats $\left.\cdot \mathrm{min}^{-1}\right)$ and time $\left(\mathrm{min} \cdot \mathrm{d}^{-1}\right)$ spent at physical activity of different intensities.

\begin{tabular}{|c|c|c|c|c|c|c|}
\hline & \multicolumn{3}{|c|}{ Off-Season } & \multicolumn{3}{|c|}{ Preseason } \\
\hline & Mean \pm SD & Min & $\operatorname{Max}$ & Mean \pm SD & Min & Max \\
\hline \multicolumn{7}{|l|}{ Heart rate } \\
\hline Rest $^{a}$ & $72 \pm 8$ & 61 & 81 & $70 \pm 4$ & 61 & 73 \\
\hline FLEX HR1 & $79 \pm 6$ & 72 & 89 & $76 \pm 3$ & 69 & 81 \\
\hline FLEX HR2 & $85 \pm 6$ & 79 & 95 & $82 \pm 6$ & 76 & 92 \\
\hline Daytime & $85 \pm 5$ & 79 & 94 & $84 \pm 5$ & 75 & 90 \\
\hline \multicolumn{7}{|l|}{ Time } \\
\hline Monitoring time & $918 \pm 42$ & 836 & 937 & $912 \pm 42$ & 846 & 948 \\
\hline Minutes > FLEX HR1 & $402 \pm 97$ & 224 & 514 & $455 \pm 102$ & 341 & 657 \\
\hline Minutes > FLEX HR2 & $297 \pm 97$ & 169 & 430 & $342 \pm 147$ & 235 & 607 \\
\hline Minutes $\geq \mathrm{HR} 50 \%$ & $39 \pm 33$ & 0 & 89 & $39 \pm 23$ & 14 & 82 \\
\hline Minutes $\geq \mathrm{HR} 70 \%$ & $12 \pm 14$ & 0 & 38 & $12 \pm 6$ & 2 & 18 \\
\hline
\end{tabular}

${ }^{a}$ Mean HR of supine, sitting, and standing during calibration.

FLEX HR1 = mean of the HRs during all resting calibration activities (i.e., supine, sitting and standing) and the lowest HR during exercising.

FLEX HR2 = mean of the highest HR during resting calibration activities (i.e., standing) and the lowest HR during exercising.

Daytime $=$ mean daytime HR during the measurement period

Minutes $\geq \mathrm{HR} 50 \%=$ time $\left(\mathrm{min}^{-1} \mathrm{~d}^{-1}\right)$ spent at an intensity level equal to $50 \%$ of $\dot{\mathrm{V}} \mathrm{O}_{2 \mathrm{max}}$.

Minutes $\geq \mathrm{HR} 70 \%=$ time $\left(\min \cdot \mathrm{d}^{-1}\right)$ spent at an intensity level equal to $70 \%$ of $\mathrm{V}_{2 \max }$.

during the off-season period. The individual increase in $\dot{\mathrm{VO}}_{2 \max }$ from the first to the second period ranged from 1.6 $\mathrm{mL} \cdot \mathrm{kg}^{-1} \cdot \mathrm{min}^{-1}$ to $7.2 \mathrm{~mL} \cdot \mathrm{kg}^{-1} \cdot \mathrm{min}^{-1}$. There was no significant difference in $\mathrm{HR}_{\max }(191.2 \pm 8.7$ vs $191.7 \pm 9.6$ bpm), maximal ventilation $(172 \pm 16.5$ vs $180.9 \pm 21.8$ $\left.\mathrm{L} \cdot \mathrm{min}^{-1}\right)$, or respiratory exchange ratio $(1.16 \pm 0.03$ vs 1.19 $\pm 0.05)$ between periods. There was a significant difference $(P<0.05)$ in postexercise maximal blood lactate between periods (off-season: $17.0 \pm 2.2$; preseason: $14.7 \pm 1.1$ $\left.\mathrm{mmol} \cdot \mathrm{L}^{-1}\right)$. The reported times spent exercising in the offseason and preseason periods, according to the training diaries, were $34 \pm 13$ and $54 \pm 19 \mathrm{~min} \cdot \mathrm{d}^{-1}(P<0.01)$, respectively.

Data on energy expenditure based on the DLW method are given in Table 2. All values were almost identical between periods, and no significant period effect was observed for any of the variables. The proportion of energy expenditure attributed to physical activity (AEE) in the off-season and preseason periods were approximately $40 \%$. The individual variation in TEE (TEE DLW) ranged from 12.8 to $25.0 \mathrm{MJ} \cdot \mathrm{d}^{-1}$ during the off-season period and from 13.9 to $23.8 \mathrm{MJ} \cdot \mathrm{d}^{-1}$ during the preseason period.

The different HR estimates and the times $\left(\min \cdot \mathrm{d}^{-1}\right)$ spent above the predetermined HR thresholds are shown in Table 3. FLEX HR1 was significantly lower than FLEX HR2 ( $P$ $=0.004)$. No significant period effect was observed on resting HR, mean daytime HR, FLEX HR1, or FLEX HR2.

A highly significant effect of subject was observed both on time spent in physical activity and on TEE $(P<0.001)$. The time spent in physical activity was significantly longer
$(P=0.02)$ when calculated from FLEX HR1 as compared with FLEX HR2. No significant period $(P=0.83)$ or method $(P=0.44)$ effect was observed on TEE based on the three respective methods, despite the significant difference between FLEX HR1 and FLEX HR2 (Table 4). TEE calculated from FLEX HR2 showed the smallest mean difference and smallest SD of differences in both periods as compared with TEE DLW (Table 4). The individual differences between TEE estimated from FLEX HR1 and TEE DLW varied from $-2.9 \%$ to $24.2 \%$ and from $-8.8 \%$ to $14.7 \%$ in the two periods, respectively, as against $-9.6 \%$ to $21.0 \%$ and -10.6 to $15.1 \%$ for the differences between FLEX HR2 and TEE DLW.

In Figure $1 \mathrm{a}-\mathrm{d}$ the difference between TEE estimated from FLEX HR1 and TEE DLW (Fig. 1, a and c) and that between FLEX HR2 and TEE DLW (Fig. 1, b and d) are plotted against the mean TEE of the methods in the respective periods. There were no significant associations between the mean of the methods and the difference between the methods for any of the plots, indicating that predicted TEE was not affected by the measurement size.

\section{DISCUSSION}

We found that TEE measured by the DLW technique and the two different estimates of TEE obtained by the FLEX HR method were similar in two periods with different training regimens in young athletes, despite a significant difference in FLEX HR as defined in the two different ways and considerable differences in the type of

TABLE 4. Total daily energy expenditure (TEE) assessed by the doubly labeled water (DLW) technique and two different FLEX HR methods in young athletes.

\begin{tabular}{|c|c|c|c|c|}
\hline & Mean \pm SD & Difference & RMS Difference & $\begin{array}{c}\text { SD of } \\
\text { Difference }\end{array}$ \\
\hline \multicolumn{5}{|l|}{ Off-season } \\
\hline TEE DLW & $16.8 \pm 3.8$ & & & \\
\hline TEE FLEX HR1 & $17.7 \pm 3.6$ & 0.9 & 2.62 & 1.38 \\
\hline TEE FLEX HR2 & $17.1 \pm 3.1$ & 0.3 & 0.72 & 1.64 \\
\hline \multicolumn{5}{|l|}{ Preseason } \\
\hline TEE DLW & $16.9 \pm 2.9$ & & & \\
\hline TEE FLEX HR1 & $17.4 \pm 2.6$ & 0.5 & 1.46 & 1.45 \\
\hline TEE FLEX HR2 & $17.0 \pm 2.7$ & 0.1 & 0.20 & 1.50 \\
\hline
\end{tabular}

Mean difference $=$ TEE FLEX HR minus TEE DLW; RMS = root mean square, calculated as square root [(sum TEE FLEX HR - TEE DLW) $\left.{ }^{2} / 8\right]$. 

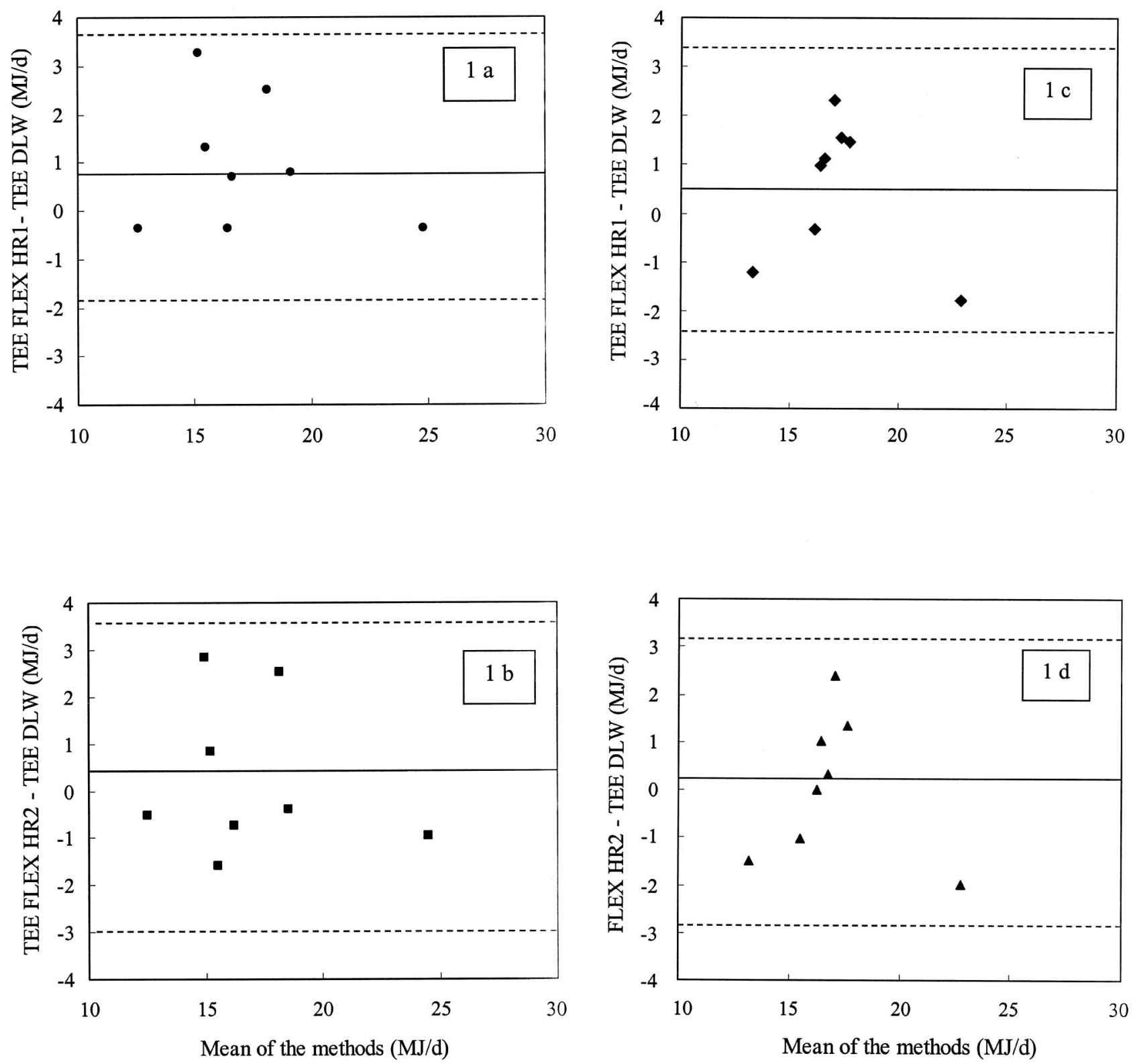

FIGURE 1-The degree of agreement between the FLEX HR method and the doubly labeled water (DLW) method for measuring total daily energy expenditure (TEE) in young athletes during two different training regimens. The mean TEE of the methods is plotted against the difference in TEE between the methods. The limits of agreement (mean difference \pm 2SD) are indicated by the dotted lines. FLEX HR1 (low FLEX HR) during off-season (a), FLEX HR2 (high FLEX HR) during off-season (b), FLEX HR1 (low FLEX HR) during preseason (c), and FLEX HR2 (high FLEX HR) during preseason (d).

physical exercises between the two study periods. The similarity in TEE between periods was also consistent with the calculated time spent in physical activity (>FLEX HR), and time spent at intensities corresponding to $50 \%$ and $70 \%$ of $\dot{\mathrm{VO}}_{2 \max }$.

The definition of the FLEX HR used to distinguish between rest and physical activity is one of the key factors with possible influence on the accuracy of the method. Livingstone et al. (11) found a close relationship $(+2 \%)$ between TEE based on FLEX HR +0 (defined as the average of the highest HR during the resting activities and the lowest HR during exercise) and TEE measured by DLW in a group of 14 adults. In contrast, Fogelholm et al. (6) concluded that, as compared with DLW-measured TEE,
FLEX HR +10 (defined as the average of the highest HR during the resting activities and the lowest HR during exercise +10 beats $\cdot \mathrm{min}^{-1}$ ) was superior to FLEX HR +0 in a group of overweight women. Another definition of the FLEX HR was used by Livingstone et al. (10) in a study of 9- to 15-yr-old children. They found no significant difference between FLEX HR estimated TEE and DLW measured TEE when defining FLEX HR as the mean of all the resting activities and the lowest HR during exercise. A similar approach was used by Heini et al. (8). They defined FLEX $\mathrm{HR}$ as the midpoint between the lowest HR during walking at $2 \mathrm{mph}$ and the HR while sitting, and found no significant difference between DLW-measured and FLEX HR-estimated TEE (8). 
The rationale for the definition of the two different FLEX HRs in the present study was based on the high aerobic fitness level of the subjects. FLEX HR +10 would consistently have been higher than the lowest HR during exercising (i.e., walking at $4.5 \mathrm{~km} \cdot \mathrm{h}^{-1}$ ) for the calibration procedures and would therefore not have represented a distinction between rest and physical activity. Furthermore, such a definition would most likely result in lower estimates of TEE.

Despite a significant difference between FLEX HR1 and FLEX HR2 and consequently a significant difference in the recorded time spent in physical activity $\left(>100 \mathrm{~min} \cdot \mathrm{d}^{-1}\right)$ between the two FLEX HR approaches, estimated TEE did not differ. This may be explained by the relatively small difference between the measured resting energy expenditure (used for all HRs $\leq$ FLEX HR) and the energy expenditure calculated from the individual regression equations during light activity (e.g., for HRs between FLEX HR1 and FLEX HR2). The mean resting energy expenditure was 6.1 $\mathrm{kJ} \cdot \mathrm{min}^{-1}$, and the average energy expenditure, calculated individually from the regression equations, corresponding to FLEX HR2 was $12.5 \mathrm{~kJ} \cdot \mathrm{min}^{-1}$. Thus, the maximal difference in energy expenditure due to the use of different definitions of the FLEX HR (and therefore to different calculations of energy expenditure for approximately 100 $\min \cdot \mathrm{d}^{-1}$ ) is close to $700 \mathrm{~kJ} \cdot \mathrm{d}^{-1}$, or approximately $4 \%$ of TEE. However, it is even more likely that the surplus in time spent in physical activity when calculated from FLEX HR1 will be spent at HRs between FLEX HR1 and FLEX HR2, and not at FLEX HR2, which will result in an even smaller difference between the two approaches. Thus, the results indicate that TEE estimated by the FLEX HR method is not affected by the choice between the two different definitions of FLEX HR used in the present study. These results corroborate the findings of Livingstone et al. (12). Although TEE was not simultaneously measured by the DLW method to verify of the accuracy of the estimated TEE in their study, there was no difference in estimated TEE even when the observed FLEX HRs ranged from $94 \pm 8$ to $111 \pm 8 \mathrm{bpm}$ with use of seven different calibration protocols in young boys (12). On the other hand, the definition of the FLEX HR will significantly affect the evaluation of the physical activity pattern (i.e., time spent above FLEX HR) if this is the aim of a HR monitoring study of this type.

Although the FLEX HR method seems to be less dependent on the definition of the FLEX HR than on the representativeness of the $\mathrm{HR}-\mathrm{VO}_{2}$ regression lines (12), it is possible that the FLEX HR is sensitive to the fitness level of the subjects. That is, in aerobically fit subjects a lower FLEX HR seems appropriate for the prediction of TEE, whereas in unfit subjects a higher FLEX HR (FLEX HR +10 ) may be more appropriate. This would be explained by the lower HR in fit subjects during light exercise (e.g., walking), which could be close to the standing HR. In some cases the standing HR may even be elevated beyond the lowest HR during exercise, as a result of an orthostatic reaction while standing. Therefore, FLEX HR +10 will probably underestimate the length of time spent in physical activity and thereby TEE in aerobically fit subjects. The reverse may be true for unfit subjects, where a lower predetermined FLEX HR may overestimate TEE if HR is elevated above the low FLEX HR as a consequence of factors other than physical activity. However, this issue needs to be further addressed in future studies.

It has been argued that the accuracy of the FLEX HR method may be improved by increasing the number of calibration activities to embrace activities associated with the normal living of the subjects $(5,9)$. In the present study, the exercises performed by the subjects, as indicated by the training diaries, differed considerably in type and duration between the two periods. During the off-season training period, all exercises were of a continuous type, predominantly running. The preseason period included both continuous and intermittent types of activities of many different forms, including weight training, in-line skating, slideboard training, skating imitations, and bicycling. Although many of these activities were not represented during the laboratory calibration, it seems that the calibration activities, which included walking, running, and bicycling at different intensities, were able to capture the free-living cardiovascular dynamics during both periods, at least on a group level. The $\mathrm{SD}$ of mean difference and root mean square difference were almost similar between periods, indicating that the calibration activities were representative of both training periods. Although the limits of agreement were far too wide for individual comparison between TEE HR and TEE DLW, with individual discrepancies ranging from approximately $-11 \%$ to $+24 \%$, it is doubtful whether the inclusion of more calibration activities would have improved the accuracy of TEE based on the FLEX HR method at an individual level.

Despite a significantly higher reported proportion of time spent in physical training during the preseason period, there were no differences in energy estimates or in time spent in physical activity of moderate and vigorous intensities $\left(>50 \% \dot{\mathrm{VO}}_{2 \max }\right)$. This could be explained by the different types of activities performed during the two periods. The amount of time reported for the training sessions during the preseason period (including intermittent exercises) was entirely recorded as the total time spent exercising, although some amount of this "training time" was spent resting between bouts. On the other hand, during the off-season period, all time spent exercising was continuous and the objectively measured time spent at $>50 \% \dot{\mathrm{VO}}_{2 \max }$ was not significantly different from the reported exercising time. Thus, a larger amount of reported time spent exercising in young athletes does not always correspond to an equally larger objectively measured amount of time in activity or to higher estimates of daily energy expenditure.

The minute-by-minute HR monitoring method was well accepted by the subjects under investigation; it is nonintrusive, and it is both simple and versatile to use during athletic training. The lack of accuracy on the individual level may be due to the highly complex nature of free-living physical activity and the associated HR-oxygen uptake dynamics. If this can be tolerated, the method seems to be well suited for 
assessing daily energy expenditure and patterns of physical activity in young athletes during different forms of training.

In summary, the results from the present study indicate that in young athletes the estimates of total energy expenditure generated by the FLEX HR method seem to be similar to those obtained by the doubly labeled water method. The agreement between the methods was not affected by the definition of the FLEX HR, and the types of training regimen seems to have only a minor impact on the estimated TEE.

\section{REFERENCES}

1. Black, A. E., W. A. Coward, T. J Cole, and A. M. Prentice. Human energy expenditure in affluent societies: an analysis of 574 doublylabelled water measurements. Eur. J. Clin. Nutr. 50:72-92, 1996.

2. Black, A. E., A. M. Prentice, and W. A. Coward. Use of food quotients to predict respiratory quotients for the doubly-labeled water method of measuring energy expenditure. Hum. Nutr. Clin. Nutr. 40:381-391, 1986.

3. Bland, J. M., and D. G. Altman. Statistical methods for assessing agreement between two methods of clinical measurement. Lancet 1(8476):307-310, 1986.

4. Boulay, M. R., O. Serresse, N. Almeras, and A. Tremblay. Energy expenditure measurements in male cross-country skiers: comparison of two field methods. Med. Sci. Sports Exerc. 26:248253, 1994.

5. Christensen, C. C., H. M. M. Frey, E. Foenstelien, E. Aadland, and H. E. Refsum. A critical evaluation of energy expenditure estimates based on individual $\mathrm{O}_{2}$ consumption/heart rate curves and average daily heart rate. Am. J. Clin. Nutr. 37:468-472, 1983.

6. Fogelholm, M., H. Hilloskorpi, R. Laukkanen, P. Oja, W. Van Marken Lichetenbelt, and K. Westerterp. Assessment of energy expenditure in overweight women. Med. Sci. Sports Exerc. 30: 1191-1197, 1998.

7. Hausswirth, C., A. X. Bigard, and J. M. Le Chevalier. The Cosmed K4 telemetry system as an accurate device for oxygen uptake measurements during exercise. Int. J. Sports Med. 18:449455, 1997.

8. Heini, A. F., G. Minghelli, E. Diaz, A. M. Prentice, and Y. Schutz. Free-living energy expenditure assessed by two different methods in rural Gambian men. Eur. J. Clin. Nutr. 50:284-289, 1996.

9. Li, R., P. Deurenberg, and J. G. A. Hautwast. A critical evaluation of heart rate monitoring to assess energy expenditure in individuals. Am. J. Clin. Nutr. 61:1146-1152, 1995.

10. Livingstone, M. B. E., W. A. Coward, A. M. Prentice, et al. Daily energy expenditure in free living children: comparison of heart rate monitoring with the doubly labelled water $\left({ }^{2} \mathrm{H}_{2}{ }^{18} \mathrm{O}\right)$ method. Am. J. Clin. Nutr. 56:343-352, 1992.
We are grateful to the speed skaters in Eskilstuna, Sweden, for participating in the study, to Olle Carlsson, Department of Statistics, Örebro University, for valuable statistical advice and to Loek Wouters, Department of Human Biology, Maastricht University, for carrying out the DLW analyses. The Public Health Committee of Stockholm's County Council and the Swedish Sports Confederation Research Council funded this project.

Address for correspondence: Ulf Ekelund, Dept. of Physical Education and Health, Örebro University, S-701 82 Örebro, Sweden; E-mail: ulf.ekelund@ioh.oru.se.

11. Livingstone, M. B. E., A. M. Prentice, A. W. Coward, et al. Simultaneous measurement of free-living energy expenditure by the doubly labeled water method and heart rate monitoring. Am. J. Clin. Nutr. 52:59-65, 1990.

12. Livingstone, M. B. E., P. J. Robson, and M. Totton. Energy expenditure by heart rate in children: an evaluation of calibration techniques. Med. Sci. Sports Exerc. 32:1513-1519, 2000.

13. Maffeis, C., Y. Schutz, L. Zoccante, L. Micciolo, and L. Pinelli. Meal-induced thermogenesis in lean and obese prepubertal children. Am. J. Clin. Nutr. 57:481-485, 1993.

14. Maffeis, C., L. Pinelli, M. Zaffanello, F. Schena, P. Iacumin, and Y. SchuTz. Daily energy expenditure in free living conditions in obese and non-obese children: comparison with doubly-labelled water $\left({ }^{2} \mathrm{H}_{2}{ }^{18} \mathrm{O}\right)$ method and heart-rate monitoring. Int. J. Obes. Relat. Metab. Disord. 19:671-677, 1995.

15. Pace, N., and E. N. Rathbun. Studies on body composition. III. The body water and chemically combined nitrogen content in relation to fat content. J. Biol. Chem. 158:685-691, 1945.

16. Schofield, W. N., C. Schofield, and W. P. T. JAmes. Predicting basal metabolic rate: new standards and review of previous work. Hum. Nutr. Clin. Nutr. 39C:5-41, 1985.

17. Spurr, G. B., A. M. Prentice, P. Murgatroyd, G. R. Goldberg, J. C. ReinA, and N. T. Christman. Energy expenditure from minute-by-minute heart-rate recording: comparison with indirect calorimetry. Am. J. Clin. Nutr. 48:552-559, 1988.

18. Thompson, J. L. Energy balance in young athletes. Int. J. Sports Nutr. 8:160-184, 1998.

19. VAn den Berg-Emons, R. J. G., W. H. M. SARis, K. R. Westerterp, and M. A. VAN BAAK. Heart rate monitoring to assess energy expenditure in children with reduced physical activity. Med. Sci. Sports Exerc. 28:496-501, 1996.

20. Westerterp, K., L. Wouters, and W. D. Van Marken LichtenBELT. The Maastricht protocol for the measurement of body composition and energy expenditure with labeled water. Obes. Res. 3(Suppl.1):49-57, 1995. 